Revue internationale P.M.E.

Économie et gestion de la petite et moyenne entreprise

\title{
Une analyse dynamique du pilotage des groupements de PME
}

\section{Sophie Peillon}

Volume 18, numéro 1, 2005

URI : https://id.erudit.org/iderudit/1008472ar

DOI : https://doi.org/10.7202/1008472ar

Aller au sommaire du numéro

\section{Éditeur(s)}

Presses de l’Université du Québec

ISSN

0776-5436 (imprimé)

1918-9699 (numérique)

Découvrir la revue

Citer cette note

Peillon, S. (2005). Une analyse dynamique du pilotage des groupements de PME. Revue internationale P.M.E., 18(1), 103-128.

https://doi.org/10.7202/1008472ar

\section{Résumé de l'article}

Constatant l'importance des coopérations entre PME, cet article s'intéresse au pilotage des groupements de PME, définis comme une forme particulière de réseau de deux PME au moins, qui s'unissent pour mener à bien un projet commun.

Pour analyser les facteurs et les moyens permettant d'assurer une efficience maximale du fonctionnement de ces groupements, l'auteur mobilise deux approches différentes mais complémentaires de la firme : la théorie contractuelle, qui insiste sur les risques inhérents aux coopérations, et la théorie basée sur les compétences, plus dynamique, qui met davantage en relief la coordination technique des activités et la possibilité de faire émerger de nouvelles compétences.

L'auteur propose une typologie opérationnelle qui distingue deux types de groupements de PME : les groupements de similitude, qui concernent des activités similaires et sont orientés vers la réduction des coûts ou la recherche d'une taille optimale, et les groupements complémentaires, fondés sur la complémentarité des actifs des partenaires et orientés vers la coproduction de ressources nouvelles. Si le premier type de groupements peut se contenter d'un pilotage contractuel basé sur des contrats " complets », le second doit s'appuyer sur un pilotage relationnel, reposant sur des contrats incomplets et la confiance, afin de favoriser les processus d'apprentissage et une coordination plus cognitive des partenaires.
Ce document est protégé par la loi sur le droit d'auteur. L'utilisation des services d'Érudit (y compris la reproduction) est assujettie à sa politique d'utilisation que vous pouvez consulter en ligne.

https://apropos.erudit.org/fr/usagers/politique-dutilisation/ 


\section{Une analyse dynamique du pilotage des groupements de $\mathrm{PME}^{\star}$}

Sophie PEILLON

École nationale supérieure des mines de Saint-Étienne

\section{MOTS CLÉS}

Compétences - Confiance - Contrats - Coopération - Groupements
Opportunisme - Pilotage - PME - Réseau

\section{RÉSUMÉ}

Constatant l'importance des coopérations entre PME, cet article s'intéresse au pilotage des groupements de PME, définis comme une forme particulière de réseau de deux PME au moins, qui s'unissent pour mener à bien un projet commun.

Pour analyser les facteurs et les moyens permettant d'assurer une efficience maximale du fonctionnement de ces groupements, l'auteur mobilise deux approches différentes mais complémentaires de la firme: la théorie contractuelle, qui insiste sur les risques inhérents aux coopérations, et la théorie basée sur les compétences, plus dynamique, qui met davantage en relief la coordination technique des activités et la possibilité de faire émerger de nouvelles compétences.

L'auteur propose une typologie opérationnelle qui distingue deux types de groupements de PME: les groupements de similitude, qui concernent des activités similaires et sont orientés vers la réduction des coûts ou la recherche d'une taille optimale, et les groupements complémentaires, fondés sur la complémentarité des

\section{L'AUTEURE}

SoPHIE PEILlon est actuellement ingénieure de recherche à l'École nationale supérieure des mines de Saint-Etienne et membre associée du Centre de recherches économiques de I'Université de Saint-Étienne (CREUSET). Détentrice d'un doctorat de sciences économiques, ses activités de recherche se centrent sur les organisations en réseau et le management des connaissances et des compétences. Adresse: École nationale supérieure des mines de Saint-Étienne, Centre G2I, 158, cours Fauriel, 42023 Saint-Étienne Cédex 2, France. Courriel: $<$ peillon@emse.fr>.

* Je tiens à remercier les évaluateurs anonymes pour leurs remarques constructives, qui m'ont permis d'améliorer le contenu de cet article.

(C) 2005 - Presses de l'Université du Québec

Édifice Le Delta I, 2875, boul. Laurier, bureau 450, Sainte-Foy, Québec G1V 2M2 • Tél.: (418) 657-4399 - www.puq.ca

Tiré de: Revue internationale P.M.E., vol. 18, $\mathrm{n}^{\circ} 1$, sous la direction de Louis Raymond $\cdot \mathrm{PME} 1801 \mathrm{~N}$

Tous droits de reproduction, de traduction et d'adaptation réservés 
actifs des partenaires et orientés vers la coproduction de ressources nouvelles. Si le premier type de groupements peut se contenter d'un pilotage contractuel basé sur des contrats "complets", le second doit s'appuyer sur un pilotage relationnel, reposant sur des contrats incomplets et la confiance, afin de favoriser les processus d'apprentissage et une coordination plus cognitive des partenaires.

\section{ABSTRACT}

Noting the importance of cooperations between SME, this paper addresses the governance of SME's groups, defined as particular shapes of networks of at least two SMEs which join together to achieve a specific project.

In order to analyze the factors and means that ensure a maximum efficiency of these groups, the author mobilizes two complementary approaches of the firm: the contractual theory, which points the inherent risks in cooperations, and the competence-based approach, which more dynamically highlights the stakes of technical coordination and the possibility of giving rise to new competencies.

The author proposes an operational typology which distinguishes two types of groups of SMEs: similarity groups are related to similar activities and directed towards a reduction of costs or the research of an optimal size; and complementary groups founded on the complementary contributions of the partners and directed towards a co-production of new resources. In the first case, a contractual governance based on complete contracts is sufficient; in the second, the governance must be relational, based on incomplete contracts and on trust, in order to support learning processes and the more cognitive coordination between the partners.

\section{RESUMEN}

Constatando la importancia de las cooperaciones entre PyMEs, este ariculo trata la gobernanza de las agrupaciones de PyMEs, definidas como una forma particular de red de dos PyMEs al menos que se unen para llevar a cabo un proyecto comun.

Para analizar los factores y medios que permiten garantizar una eficiencia maxima del funcionamiento de estas agrupaciones, el autor moviliza dos enfoques diferentes pero complementarios de la empresa: la teoria contractual, que insiste sobre los riesgos inherentes de las cooperaciones, y la teoria dicha "competencebased approach», mas dinamica, que pone aun mas de relieve la coordinación tecnica entre los socios y la posibilidad de hacer surgir nuevas competencias.

El autor propone una tipología opertiva que distingue dos tipos de agrupaciones de PyMEs: las agrupaciones e semejanza, que se refieren a actividades similares, orientadas hacia la reducción de los costes o la busqueda de un tamano optimo: y las agrupaciones complementarias, fundadas sobre la complementariedad de los activos de los socios y orientadas hacia la coproducción de nuevos recursos. Si el primer tipo de agrupaciones puede satisfacerse con una gobernanza contractual basada en contratos mas completos, el segundo debe basarse en una gobernanza relacional, fundada en contratos incompletos y confianza, con el fin de favorecer los procesos de aprendizaje y una coordinación mas cognoscitiva de los socios.

Revue internationale P.M.E., vol. 18, $\mathrm{n}^{\circ} 1,2005$

(C) 2005 - Presses de l'Université du Québec

Édifice Le Delta I, 2875, boul. Laurier, bureau 450, Sainte-Foy, Québec G1V 2M2 - Tél.: (418) 657-4399 - www.puq.ca

Tiré de: Revue internationale P.M.E., vol. 18, $\mathrm{n}^{\circ} 1$, sous la direction de Louis Raymond • PME1801N

Tous droits de reproduction, de traduction et d'adaptation réservés 


\section{ZUSAMMENFASSUNG}

Der Artikel umschreibt die Wichtigkeit und Bedeutung von Kooperationen unter KMUs und legt besonderes Augenmerk auf die Führung dieser KMU-Gruppierungen. Diese Gruppierungen werden definiert als spezielles Netzwerk (mindestens zwei Partner) mit dem Ziel, ein gemeinsames Vorhaben erfolgreich durchzuführen.

Um die Faktoren und Mittel zu analysieren, die eine bestmögliche Effizienz solcher Gruppierungen erlauben, werden zwei verschiedene sich ergänzende Ansätze herangezogen: die Vertragstheorie, wonach Risiken in solchen Zusammenarbeiten inhärent sind und der dynamischeren Kompetenztheorie, die besonders die technische Koordination und die Möglichkeit neuer Kompetenzentwicklungen hervorhebt.

Der Autor schlägt eine operationelle Typologie vor, die zwei Arten von KMUGruppierungen unterscheidet: Gruppierungen von ähnlichen Unternehmen und ähnlichen Aktivitäten, die eine Kostenreduktion oder eine kritische Grösse anstreben, und Gruppierungen, in welchen sich die einzelnen Partner ergänzen und eine Koproduktion von neuen Ressourcen anstreben. Für die erstgenannte Gruppierung benötigt man zur Steuerung lediglich ein komplettes Vertragswerk. Die zweite Gruppierung bedarf zur eigenen Leitung flexible Rahmenverträge und Vertrauen, um Lernprozesse und eine kognitive Koordination der Partner zu begünstigen.

\section{Introduction}

Les années 1980 et 1990 sont aujourd'hui largement reconnues comme ayant été l'ère des coopérations interfirmes. On assiste en effet alors à une multiplication du nombre d'accords de coopération ${ }^{1}$, aux plans national et international, sous la pression à la fois de la globalisation des économies et de la complexité croissante du développement technologique.

Ainsi, le recours à la coopération s'impose dans les années 1980 comme l'un des outils privilégiés par les grandes entreprises pour obtenir de nouvelles parts de marché ou accéder à de nouvelles technologies. Dans les années 1990, les PME leur emboîtent le pas et commencent elles aussi à envisager la coopération comme une stratégie de développement, voire, pour certaines, de survie ${ }^{2}$.

En effet, les PME ont également subi les modifications du contexte industriel, touchées soit «par ricochet» pour les PME sous-traitantes, les nouvelles contraintes de productivité se répercutant tout au long de la chaîne de production verticale - les fournisseurs de premier rang devenant de plus en plus exigeants avec leurs propres

1. Et à leur médiatisation accrue, qui renforce l'impression de leur développement.

2. Si nous utilisons le terme générique de PME, notre analyse concerne en fait davantage les PMI. En effet, comme les exemples l'illustreront, les cas que nous avons étudiés sont issus du secteur industriel et non des secteurs agricole ou des services.

Revue internationale P.M.E., vol. 18, n 1, 2005

(C) 2005 - Presses de l'Université du Québec

Édifice Le Delta I, 2875, boul. Laurier, bureau 450, Sainte-Foy, Québec G1V 2M2 - Tél.: (418) 657-4399 - www.puq.ca

Tiré de: Revue internationale P.M.E., vol. 18, $\mathrm{n}^{\circ} 1$, sous la direction de Louis Raymond • PME1801N

Tous droits de reproduction, de traduction et d'adaptation réservés 
fournisseurs, et ainsi de suite -, soit directement pour les PME présentes sur le marché final, subissant les mêmes contraintes que les grandes entreprises - exacerbation de la concurrence, accélération du progrès technologique, remise en question des mécanismes du marché concurrentiel et évolution des conditions de l'échange.

C'est dans ce contexte que le phénomène de «groupement de PME» semble se développer ${ }^{3}$. Le groupement recouvre des formes très variées de coopération; mais, globalement, il s'agit de plusieurs PME (généralement plus de deux) qui se regroupent, sous des formes juridiques variées (association, groupement d'intérêt économique, création d'une entreprise conjointe, etc.), tout en restant juridiquement indépendantes, afin de mener à bien un projet, pouvant aller du simple partage d'une ressource jusqu'à la réalisation d'une innovation en commun ${ }^{4}$.

Sur le plan conceptuel, le groupement constitue une forme de coopération et se situe donc «entre» les deux formes polaires d'organisation que sont l'entreprise - reposant sur des relations d'ordre hiérarchique - et le marché, fondé sur le mécanisme des prix. En effet, le groupement est différent de la hiérarchie, dans la mesure où, dans cette dernière, il n'existe pas une pluralité de centres de décision autonomes (pouvant prendre des décisions conjointement). Dans l'organisation hiérarchique, la direction détient in fine le pouvoir de décider; elle est le «centre ultime de recours» en cas de conflit entre les différentes entités de l'organisation. Le groupement est donc distinct d'une fusion-acquisition. Mais il ne peut pas non plus être considéré comme une organisation strictement marchande puisque la médiatisation des relations entre les agents n'y est pas assurée uniquement par les prix.

La question que l'on peut dès lors se poser est celle des mécanismes de coordination interentreprises au sein des groupements, c'est-à-dire du «pilotage»

3. Si les coopérations entre PME existent depuis longtemps, il semble en effet que la tendance au regroupement de PME se soit accentuée depuis le milieu des années 1990. Il n'existe pas de statistiques précises sur la coopération entre PME, car c'est un phénomène moins médiatisé que la coopération entre grandes firmes et difficile à repérer et à évaluer, mais les acteurs de terrain en contact avec le milieu industriel (institutions locales telles que les chambres de commerce et d'industrie), eux-mêmes relayés par les publications orientées vers l'industrie se font de plus en plus l'écho de pratiques de ce type. De plus, de nombreux gouvernements, en particulier en Europe, ont mis en œuvre des politiques visant à favoriser ce type de coopération (l'Italie, le Danemark, l'Allemagne ou l'Espagne; OCDE, 1996).

4. Notons d'ores et déjà qu'il convient de distinguer les groupements dont nous parlons ici des « groupements momentanés d'entreprises », définis comme une agrégation temporaire de compétences et de ressources qui collaborent ensemble pour un besoin spécifique et limité dans le temps, telle une opportunité d'affaires.

Revue internationale P.M.E., vol. 18, nº 1, 2005

(C) 2005 - Presses de l'Université du Québec

Édifice Le Delta I, 2875, boul. Laurier, bureau 450, Sainte-Foy, Québec G1V 2M2 • Tél.: (418) 657-4399 - www.puq.ca

Tiré de: Revue internationale P.M.E., vol. 18, $\mathrm{n}^{\circ}$ 1, sous la direction de Louis Raymond • PME1801N

Tous droits de reproduction, de traduction et d'adaptation réservés 
des groupements de PME. Par pilotage, nous entendons les moyens mis en œuvre par les entreprises partenaires pour coordonner efficacement leurs actions et leurs décisions au sein de la coopération.

La question du pilotage des coopérations n'est évidemment pas propre aux PME-PMI, mais se pose aux entreprises qui coopèrent, quelle que soit leur taille. Aussi, le modèle conceptuel que nous proposons est-il de portée générale. Cependant, dans le cadre d'une approche de nature constructiviste, largement inspirée de la réalité de terrain, nous avons souhaité l'appliquer plus spécifiquement aux PME-PMI, car peu de travaux ont été consacrés à l'étude de la coordination au sein des groupements de PME, en dehors des analyses à base territoriale (comme celles concernant les districts industriels ou les systèmes productifs locaux).

Nous commencerons par définir le groupement; nous verrons qu'il s'agit d'une forme de réseau, et que deux grands types de groupements peuvent être distingués: des groupements de similitude et des groupements complémentaires. Ensuite, en nous inspirant des théories de la firme, nous verrons que le pilotage consiste à mettre en œuvre un ensemble de modes de coordination permettant aux partenaires de la coopération de limiter les risques potentiels qu'ils encourent et de maximiser les bénéfices qu'ils sont susceptibles d'en retirer. Une distinction entre deux types de pilotage, contractuel et relationnel, sera alors proposée. Nous pourrons alors montrer qu'à chaque type de groupement correspond un mode de pilotage adéquat: contractuel, pour les groupements de similitude, et relationnel, pour les groupements complémentaires.

\section{Le cadre d'analyse: la coopération au sein des groupements de PME}

\subsection{Coopération interfirmes et groupements de PME: une mise en perspective}

Nous avons défini le groupement simplement comme s'agissant de plusieurs PME qui se regroupent afin de mener à bien un projet tout en restant juridiquement indépendantes. Cette définition laisse entrevoir l'extrême diversité du phénomène: le groupement de PME est, à l'image de la coopération interfirmes, un phénomène à la fois particulier et multiforme; les formes prises par l'accord, les objectifs visés et les PME concernées varient considérablement selon les cas. Dès lors, il convient de définir précisément la coopération au sein des groupements.

\section{Le groupement: une forme de réseau d'entreprises}

La coopération ayant fait l'objet d'un nombre extrêmement important de travaux, il règne un réel éclectisme dans les qualificatifs utilisés, les formes et les objectifs

Revue internationale P.M.E., vol. 18, nº 1, 2005

(C) 2005 - Presses de l'Université du Québec

Édifice Le Delta I, 2875, boul. Laurier, bureau 450, Sainte-Foy, Québec G1V 2M2 - Tél.: (418) 657-4399 - www.puq.ca

Tiré de: Revue internationale P.M.E., vol. 18, $\mathrm{n}^{\circ} 1$, sous la direction de Louis Raymond • PME1801N

Tous droits de reproduction, de traduction et d'adaptation réservés 
relevés et il n'existe pas de définition unanime de la coopération interfirmes, qui recouvre elle-même divers types d'accords ${ }^{5}$. De plus, bien que de nombreux travaux existent sur la coopération entre petites entreprises, notamment au sein des districts industriels ou des systèmes productifs locaux, il n'existe à notre connaissance pas de littérature sur les groupements de PME.

Après avoir passé en revue une grande partie de la littérature sur la coopération entre firmes et entre PME, il nous semble que la forme de coopération la plus appropriée pour caractériser le groupement est celle de réseau. En effet, le groupement peut être conçu comme une forme de réseau de firmes, possédant des caractéristiques propres: le groupement est un réseau composé d'au moins deux PME indépendantes sur le plan juridique (et généralement plus), qui s'unissent pour une certaine durée afin de mener à bien un projet commun nécessitant une coordination de leurs comportements. La coopération au sein du réseau peut être ou non territorialisée ${ }^{6}$, peut être de type vertical et/ou horizontal ou intersectoriel ${ }^{7}$, mais comporte dans tous les cas une dimension formelle, allant de la signature d'un contrat jusqu'à la création d'une entreprise conjointe.

Cette définition, quoique nécessaire, nous semble cependant insuffisante pour analyser le pilotage des groupements. En effet, pour cela, il convient de définir précisément la nature même de la coopération. C'est pourquoi nous proposons une typologie des groupements en fonction de l'objectif poursuivi par les partenaires.

\section{Groupement de similitude et groupement complémentaire}

Il est possible de montrer qu'il existe deux principaux types de groupements selon l'objectif de la coopération. En effet, de cet objectif découle la nature des activités qui doivent être mises en œuvre afin de parvenir à la réalisation du projet commun aux partenaires. Ces activités pouvant être soit similaires, soit complémentaires, nous définirons les groupements comme étant soit des «groupements de similitude», soit des «groupements complémentaires».

La première catégorie de motivations au regroupement renvoie à une logique de réduction des coûts et de recherche d'économies d'échelle, passant généralement par le partage de ressources et/ou la centralisation de fonction. L'objectif est alors d'atteindre une «taille optimale» dans la production ou d'autres fonctions

5. Rappelons que de très nombreux travaux ont été réalisés sur le management des alliances, notamment selon une perspective réseau et basée sur les compétences ( $c f$. par exemple Duysters, de Man et Wildeman, 1999; Duysters et Heimeriks, 2002).

6. Ce qui distingue les groupements des districts industriels, des réseaux locaux d'innovation ou des systèmes productifs locaux.

7. Du fait de ses multiples formes, le groupement ne peut être complètement assimilé à un partenariat vertical ou à une alliance horizontale (Peillon, 2001).

Revue internationale P.M.E., vol. 18, nº 1, 2005

(C) 2005 - Presses de l'Université du Québec

Édifice Le Delta I, 2875, boul. Laurier, bureau 450, Sainte-Foy, Québec G1V 2M2 • Tél.: (418) 657-4399 - www.puq.ca

Tiré de: Revue internationale P.M.E., vol. 18, $\mathrm{n}^{\circ}$ 1, sous la direction de Louis Raymond • PME1801N

Tous droits de reproduction, de traduction et d'adaptation réservés 
(p. ex., les achats), la mise en commun de certaines activités ou des investissements communs permettant de partager et de réduire les coûts. La coopération répond ici à une volonté d'économiser les moyens et va concerner des activités similaires, que Richardson (1972, p. 888) définit comme requérant des capacités identiques ou intimement liées pour être exercées. Les apports de chaque partenaire au groupement sont similaires; il peut s'agir d'entreprises centralisant une fonction commune, par exemple les achats ou la gestion des stocks, ou partageant un investissement, notamment une ressource humaine.

La seconde catégorie de motivations correspond à une logique de recherche d'opportunités nouvelles, commerciales ou technologiques. Il s'agit de conquérir de nouveaux marchés, grâce à la proposition d'une offre globale ou d'une innovation commune. La stratégie peut être considérée ici comme plus «offensive» et l'objectif global est alors de rapprocher et renforcer des compétences par une recherche de synergie entre les partenaires. Les activités qui entrent dans le processus de coopération sont donc nécessairement complémentaires, correspondant à différentes phases d'un processus de production au sens large ${ }^{8}$. Il s'agit, par exemple, d'entreprises cherchant à développer conjointement une innovation ou se regroupant pour proposer une offre globale.

Cette distinction avait déjà été mise en exergue par Joffre et Koenig dans les années 1980. En effet, ces auteurs avaient alors caractérisé deux types fondamentaux de coopération: dans une «coopération de similitude», des entreprises ayant des problèmes identiques groupent des moyens de même nature pour en économiser l'usage ou pour atteindre une masse critique autrement inaccessible (dans le domaine commercial ou en R-D par exemple); alors que la «coopération de différence» repose sur une «combinaison de complémentarités» autorisant «une utilisation plus complète ou plus intense d'actifs détenus de manière dissymétrique» (Joffre et Koenig, 1984, p. 67). Nous reprenons donc cette distinction, car elle nous semble particulièrement pertinente en ce qui concerne les groupements de $\mathrm{PME}^{9}$.

8. Richardson définit un processus de production « au sens large » comme étant « par exemple, non seulement les relations entre la construction des automobiles et celle de leurs composants mais encore la relation de chacune d'elles aux activités correspondantes de R-D et de vente » (1972, p. 889).

9. Notons cependant que les deux catégories de groupements ne sont pas parfaitement étanches: généralement, le groupement poursuit plusieurs objectifs à la fois; il est donc extrêmement difficile de dégager un objectif unique pour chaque groupement. De plus, le choix de se regrouper ne correspond pas nécessairement à une stratégie identique de la part de toutes les entreprises qui y participent.

Revue internationale P.M.E., vol. 18, n 1, 2005

(C) 2005 - Presses de l'Université du Québec

Édifice Le Delta I, 2875, boul. Laurier, bureau 450, Sainte-Foy, Québec G1V 2M2 - Tél.: (418) 657-4399 - www.puq.ca

Tiré de: Revue internationale P.M.E., vol. 18, $\mathrm{n}^{\circ} 1$, sous la direction de Louis Raymond $\cdot$ PME1801N

Tous droits de reproduction, de traduction et d'adaptation réservés 


\subsection{Le pilotage des coopérations interfirmes}

L'identification des modalités de pilotage des coopérations interfirmes requiert, selon nous, de procéder en deux étapes: la première consiste à relever les problèmes qui peuvent se poser aux entreprises dans le cadre de leur coopération; la seconde, à proposer des mécanismes leur permettant de résoudre ou de limiter ces problèmes. Pour cela, il est nécessaire de nous attarder aux théories récentes de la firme.

\section{La complémentarité des approches contractuelles et basées sur les compétences}

La coordination est une question centrale de l'analyse économique. De très nombreux travaux ont été consacrés aussi bien à la coordination interindividuelle qu'à la coordination interfirmes. De manière très générale, ces analyses tentent de comprendre comment, conscients de leurs interdépendances et de leurs divergences d'intérêt, les agents économiques (individus ou firmes) mettent en place des dispositifs destinés à rendre leurs interactions efficientes.

En schématisant, il est possible de distinguer deux groupes au sein de ces théories; certaines sont centrées sur les questions relevant des motivations des agents et sont préoccupées par le risque de comportement opportuniste auquel chaque agent est soumis de la part des autres, alors que d'autres se focalisent sur les caractéristiques «techniques» de la coopération. On retrouve ici la distinction bien connue entre approches contractuelles et approches basées sur les compétences.

Même s'ils ont très souvent été opposés, ces deux paradigmes nous semblent complémentaires pour analyser la coordination au sein des groupements de $\mathrm{PME}^{10}$. En effet, chacun tient pour donné ce que l'autre questionne ou cherche à démontrer. Les approches contractuelles, qui s'intéressent aux conflits d'intérêt, considèrent les bienfaits «techniques» de la coopération comme donnés; les approches basées sur les compétences, centrées sur les difficultés et potentialités «techniques» de la coopération, considèrent les conflits comme résolus.

Or, un groupement est certes une entité dont la raison d'être est de mener à bien un projet ou une activité - il repose donc sur la coopération des partenaires; mais les membres du groupement restent indépendants et conservent donc des préférences, des intérêts et des objectifs propres, au caractère potentiellement conflictuel. En transposant leur analyse au groupement de PME, on peut dire que les approches contractuelles conduisent à s'interroger sur la vulnérabilité de chaque

10. Les partisans du premier reprochent le manque de formalisme du second; ceux du second déplorent l'incapacité du premier à prendre en compte le changement technologique. Mais notre propos n'est pas ici d'entrer dans un débat d'ordre analytique et épistémologique concernant la pertinence de ces deux visions de l'organisation industrielle.

Revue internationale P.M.E., vol. 18, nº 1, 2005

(C) 2005 - Presses de l'Université du Québec

Édifice Le Delta I, 2875, boul. Laurier, bureau 450, Sainte-Foy, Québec G1V 2M2 • Tél.: (418) 657-4399 - www.puq.ca

Tiré de: Revue internationale P.M.E., vol. 18, $\mathrm{n}^{\circ}$ 1, sous la direction de Louis Raymond PME1801N

Tous droits de reproduction, de traduction et d'adaptation réservés 
partenaire du groupement vis-à-vis des autres et sur les façons de remédier à ce risque d'opportunisme ${ }^{11}$. Les approches fondées sur les compétences, quant à elles, amènent à étudier dans quelle mesure le groupement peut permettre une amélioration des performances productives des participants. Une perspective fondée sur les compétences conduit à considérer que chaque membre accepte de travailler dans l'intérêt du groupement, alors qu'une perspective contractuelle consiste à tenter de cerner les dispositifs permettant d'aboutir ou de se rapprocher d'un tel comportement. Les deux types d'approches doivent donc être mobilisés afin d'aboutir à une vision globale du pilotage du groupement, comme consistant non seulement à en «minimiser» les risques, mais aussi à en «maximiser» les bénéfices.

\section{Les deux enjeux du pilotage des coopérations interfirmes}

Sur la base d'une synthèse entre les deux perspectives évoquées, nous proposons de distinguer deux enjeux essentiels du pilotage des coopérations interfirmes: le premier consiste à limiter les conflits d'intérêts potentiels, c'est-à-dire le risque d'opportunisme sur lequel sont centrées les théories des contrats; le second consiste à maîtriser les difficultés et à exploiter les potentialités techniques des coopérations, notamment en termes de création de compétences, ce que nous appelons la «coordination technique ${ }^{12} »$ et sur laquelle se centrent les approches basées sur les compétences.

\section{La gestion de l'opportunisme}

Une approche contractuelle de la coopération interfirmes conduit à insister sur le rôle des asymétries contractuelles et particulièrement de l'opportunisme dans la coordination des entreprises partenaires. En effet, une grande partie des difficultés auxquelles se heurte la coordination provient de l'incertitude comportementale, qui est réelle parce que les partenaires sont dotés d'une rationalité limitée et que la coopération entre entreprises est une situation dans laquelle les contrats sont généralement incomplets. Comme les intérêts individuels des parties ne sont pas nécessairement compatibles, la recherche de l'intérêt personnel peut nuire à l'efficacité de la coopération. De ce fait, l'incertitude comportementale qui règne autour des transactions est inévitable.

11. Il s'agit, par exemple, de traiter du dilemme entre apporter suffisamment de ressources pour que la coopération soit forte et ne pas en apporter trop pour que le partenaire puisse trop se renforcer.

12. En référence à Brousseau (1993).

Revue internationale P.M.E., vol. 18, nº 1, 2005

(C) 2005 - Presses de l'Université du Québec

Édifice Le Delta I, 2875, boul. Laurier, bureau 450, Sainte-Foy, Québec G1V 2M2 - Tél.: (418) 657-4399 - www.puq.ca

Tiré de: Revue internationale P.M.E., vol. 18, $\mathrm{n}^{\circ} 1$, sous la direction de Louis Raymond • PME1801N

Tous droits de reproduction, de traduction et d'adaptation réservés 
C'est pourquoi le risque d'opportunisme ex post ${ }^{13}$, qui est aggravé par la présence d'actifs spécifiques à la relation de coopération, apparaît comme l'un des problèmes majeurs auxquels sont confrontés les partenaires. Le premier enjeu du pilotage de la coopération est donc d'éviter l'apparition de comportements opportunistes, nuisibles à l'efficacité de la coordination.

\section{La coordination technique}

La coordination technique concerne la prise de décision au sein de la coopération devant permettre l'allocation des ressources initiales et la division des tâches entre les partenaires mais aussi la création de ressources nouvelles. La coordination technique consiste non seulement à maîtriser les problèmes d'information, mais aussi à régler les transferts de connaissances et à faciliter la création de compétences nouvelles, donc l'apprentissage. Il s'agit d'assurer une prise de décision efficace malgré la rationalité au moins limitée des partenaires et l'incertitude environnementale et de favoriser l'apprentissage entre partenaires, en dépit de la nature tacite et organisationnelle des connaissances et compétences.

Selon nous, il existe en fait deux manières d'affronter ces problèmes d'opportunisme et de coordination technique.

\section{Pilotage contractuel et pilotage relationnel}

La première consiste à s'appuyer sur les contrats et à mettre en œuvre un pilotage «contractuel». Au début de la relation, les partenaires s'engagent sur un contrat relativement complet, qui prévoit un ensemble de mécanismes de gestion de l'opportunisme et de coordination technique ${ }^{14}$ et, par la suite, le pilotage sera «automatique», au sens où les partenaires se borneront à appliquer ce qui a été prévu dans le contrat de coopération.

Cependant, d'une part, cette forme de pilotage requiert un environnement stable et, d'autre part, les contrats sont coûteux, d'une efficacité limitée et inadaptés en ce qui concerne les processus d'apprentissage. Une deuxième solution consiste

13. L'aléa moral peut conduire les partenaires à adopter des comportements de free rider, c'est-à-dire «tirer au flanc », surexploiter une ressource commune ou encore tenter un hold-up de la quasi-rente issue de leur coopération.

14. Concernant la gestion de l'opportunisme, le pilotage va consister à s'assurer que les agents impliqués dans la coopération participent au projet commun, à la fois en fournissant les informations appropriées et en agissant conformément aux objectifs de la coopération. Concernant la coordination technique, des règles de comportement et un mécanisme d'autorité vont être prévus ex ante.

Revue internationale P.M.E., vol. 18, nº 1, 2005

(C) 2005 - Presses de l'Université du Québec

Édifice Le Delta I, 2875, boul. Laurier, bureau 450, Sainte-Foy, Québec G1V 2M2 • Tél.: (418) 657-4399 - www.puq.ca

Tiré de: Revue internationale P.M.E., vol. 18, $\mathrm{n}^{\circ}$ 1, sous la direction de Louis Raymond • PME1801N

Tous droits de reproduction, de traduction et d'adaptation réservés 
alors à mettre en œuvre un pilotage «relationnel». Dans ce cas, les partenaires vont signer un contrat largement incomplet, qui sera complété par d'autres mécanismes, tels que la confiance, construits par les acteurs dans le cours de leur relation.

Autrement dit, l'objectif d'un pilotage contractuel est de parvenir à un contrat de coopération qui soit le plus complet possible, alors qu'un pilotage relationnel va laisser le contrat incomplet et faire appel à d'autres mécanismes complémentaires (confiance, ajustement mutuel, etc. ${ }^{15}$. Ainsi, dans une perspective statique en termes d'allocation des ressources, le pilotage de la coopération consistera à définir ex ante des moyens de coordination efficaces. L'enjeu est alors une simple «mise en cohérence des comportements individuels » des partenaires grâce à des mécanismes contractuels prédéfinis. Dans une perspective plus dynamique, le pilotage de la coopération nécessite non seulement une cohérence des comportements individuels au niveau allocatif qui doit de plus être robuste et durable, mais surtout la création d'une véritable «capacité collective de production et d'organisation ${ }^{16}$ » dans un objectif de création de ressources ${ }^{17}$.

Après avoir précisé ce cadre d'analyse, nous pouvons maintenant revenir aux groupements de PME.

\section{Le pilotage des groupements de PME}

Le groupement nécessite un mode de pilotage particulier, car il diffère de la hiérarchie et du marché. D'une part, la coordination des activités des partenaires doit être efficace pour espérer atteindre les objectifs fixés, réaliser le projet commun et optimiser les bénéfices du regroupement. D'autre part, les entités participant au groupement restent juridiquement autonomes: si le groupement est formé pour atteindre un objectif supposé commun, cela n'empêche pas chaque PME de conserver des objectifs propres. Il peut donc exister des conflits d'intérêts et le risque d'opportunisme est effectivement présent au sein des groupements de PME.

Ainsi, le pilotage des groupements consiste à assurer à la fois la coordination technique et la gestion des intérêts entre les différents partenaires. L'analyse de ce pilotage ne peut selon nous qu'être dynamique. En effet, une des caractéristiques du groupement est d'engendrer des relations interentreprises durables, ce qui

15. Remarquons que les prix constituent un élément important de la coordination interentreprises, dans le pilotage contractuel comme dans le pilotage relationnel.

16. Ces deux expressions nous ont été inspirées par Weinstein (1997, p. 410).

17. Sur les notions de pilotage contractuel et relationnel, le lecteur peut se reporter notamment à Poppo et Zenger (2002).

Revue internationale P.M.E., vol. 18, n 1, 2005

(C) 2005 - Presses de l'Université du Québec

Édifice Le Delta I, 2875, boul. Laurier, bureau 450, Sainte-Foy, Québec G1V 2M2 • Tél.: (418) 657-4399 - www.puq.ca

Tiré de: Revue internationale P.M.E., vol. 18, $\mathrm{n}^{\circ} 1$, sous la direction de Louis Raymond $\cdot$ PME1801N

Tous droits de reproduction, de traduction et d'adaptation réservés 
explique de prendre en compte la dimension temporelle de la coopération. Pour ce faire, nous considérerons que le processus de coopération peut être décomposé en trois phases ${ }^{18}$ :

- à la phase d'engagement, la négociation aboutit à la signature du contrat de coopération par les partenaires;

- durant la phase de réalisation, le processus de coopération est effectivement mis en œuvre;

- enfin, lors de la phase de cessation de la coopération, le groupement peut évoluer vers l'une des trois formes organisationnelles possibles: un renouvellement de la coopération, un retour à des relations marchandes ou une intégration des partenaires.

Nous examinons ci-après chacune de ces trois phases du processus de coopération pour chaque type de groupement identifié, ce qui nous conduit à montrer que le pilotage des groupements de PME est sensiblement différent selon que le groupement est de similitude ou complémentaire: un pilotage contractuel semble convenir aux groupements de similitude, alors qu'un pilotage relationnel paraît plus adapté aux groupements complémentaires.

\subsection{Les groupements de similitude: un pilotage essentiellement contractuel}

\section{Des enjeux aisément circonscrits}

Dans le cas des groupements de similitude, les PME groupent des moyens de même nature pour en économiser l'usage et/ou atteindre une taille optimale. On retrouve dans cette catégorie les coopérations axées sur le partage d'une ressource, humaine ou matérielle, ou la centralisation de fonctions particulières, afin d'obtenir des économies d'échelle. Cela donne alors la possibilité aux entreprises, soit de diminuer leurs coûts de fonctionnement, soit d'avoir accès à des ressources qui sont nécessaires à leur activité mais dont elles n'ont pas besoin à temps plein. Par exemple, plusieurs firmes peuvent partager le coût d'un département des ventes pour promouvoir conjointement leurs produits, combiner leur capacité productive pour offrir aux clients la possibilité de satisfaire de plus grosses commandes, combiner leur pouvoir d'achat pour s'assurer de meilleures conditions d'approvisionnement, utiliser un programme de formation commun pour leurs personnels, acheter ensemble et partager un équipement coûteux, etc.

18. Nous nous inspirons de plusieurs auteurs, en particulier Ring et Van de Ven (1994), Doz (1996) et Llerena, Matt et Wolff, 1999.

Revue internationale P.M.E., vol. $18, \mathrm{n}^{\circ} 1,2005$

(C) 2005 - Presses de l'Université du Québec

Édifice Le Delta I, 2875, boul. Laurier, bureau 450, Sainte-Foy, Québec G1V 2M2 • Tél.: (418) 657-4399 - www.puq.ca

Tiré de: Revue internationale P.M.E., vol. 18, $\mathrm{n}^{\circ}$ 1, sous la direction de Louis Raymond • PME1801N

Tous droits de reproduction, de traduction et d'adaptation réservés 


\section{Une coordination technique relativement aisée}

L'activité commune est bien délimitée et ne nécessite pas de combinaison de ressources ou de compétences distinctes et, par conséquent, implique peu d'interactions entre les partenaires. Le degré d'incertitude est faible et les modalités d'intervention des différentes parties peuvent donc être clairement définies. La coordination technique est peu problématique: il n'existe que peu d'incertitude sur le résultat final et les moyens à mettre en ouvre. Elle va par exemple consister à déterminer la répartition d'une commande enregistrée par le groupement entre les différentes PME, ou les modalités d'utilisation d'une ressource commune par les partenaires.

\section{Un risque d'opportunisme présent mais limité}

Puisque la coopération ne requiert finalement pas de travail réellement commun, les problèmes d'opportunisme se résument le plus souvent à un risque de surexploitation d'une ressource commune.

En effet, souvent, les PME forment un groupement afin d'acheter en commun une machine coûteuse ou partager un salarié dont elles n'auraient pu supporter le coût seules. Dans ce cas, le groupement nécessite de réaliser un investissement spécifique, par exemple, lorsqu'il s'agit d'acheter un équipement à plusieurs pour en partager les coûts. L'actif étant spécifique, les PME sont dans une situation de relative dépendance les unes par rapport aux autres, ce qui vient, en théorie, renforcer le risque d'opportunisme: il est possible, du fait d'une propriété commune de la ressource, que certains partenaires en profitent pour ne pas l'entretenir ou abuser de son usage. Ainsi, les entreprises du groupement peuvent avoir intérêt à mobiliser davantage un salarié, à utiliser davantage un équipement au détriment des partenaires, ou à être réticentes à investir dans la formation du personnel, dans l'entretien et la maintenance d'un équipement, puisqu'elles n'en assument pas entièrement les coûts et qu'elles n'en retirent pas tous les bénéfices.

\section{Un pilotage essentiellement contractuel}

Dans la plupart des cas, un pilotage contractuel peut, selon nous, tout à fait convenir à ce type de groupement.

\section{La phase d'engagement: un contrat de coopération «complet»}

À la phase d'engagement, les partenaires s'accordent sur un certain nombre de règles de comportement, spécifiées dans le contrat. Par exemple, le contrat va déterminer une règle de répartition d'une commande entre les partenaires lors d'une production commune (par exemple, au prorata du chiffre d'affaires de chaque

Revue internationale P.M.E., vol. 18, n 1, 2005

(C) 2005 - Presses de l'Université du Québec

Édifice Le Delta I, 2875, boul. Laurier, bureau 450, Sainte-Foy, Québec G1V 2M2 - Tél.: (418) 657-4399 - www.puq.ca

Tiré de: Revue internationale P.M.E., vol. 18, $\mathrm{n}^{\circ} 1$, sous la direction de Louis Raymond PME1801N

Tous droits de reproduction, de traduction et d'adaptation réservés 
membre) ou les modalités d'utilisation d'une ressource commune (par exemple, un certain nombre d'heures par jour). Le problème de la surexploitation d'une ressource commune peut généralement être évité grâce à des règles contractuelles définissant ex ante les modalités d'utilisation et d'entretien de l'actif par chaque partenaire. Au total, le contrat (formalisé ou tacite) qui précise les arrangements entre les entreprises lors du travail en coopération est relativement complet.

La mise en œuvre de la coopération: un potage «automatique»

Les mécanismes de coordination pouvant être presque complètement définis à la phase d'engagement, la coopération peut être pilotée «automatiquement», grâce aux mécanismes prévus dans le contrat. L'activité commune est réalisée selon les modalités arrêtées dans le contrat et la ressource commune est utilisée conformément aux prescriptions contractuelles. Si des conflits surviennent, les partenaires ont recours aux solutions prévues au début de la relation. Le partage des résultats est lui aussi prévu ex ante.

\section{Cessation et évolution des groupements de similitude}

Quant à la cessation de la coopération, elle peut également être prévue ex ante: le groupement est dissous lorsque l'objectif est atteint, les PME retrouvant ensuite de simples relations de marché.

Au total, l'échéance et le but sont définis préalablement, ce qui permet de donner des objectifs concrets et temporels précis. Les missions de chacun sont définies ainsi que l'organisation du travail; sont également fixées les règles de rémunération.

Les conditions de fonctionnement d'un groupement de similitude sont décrites d'abord par le contrat: les conditions du contrat qui lient les partenaires sont prépondérantes.

\subsection{Les groupements complémentaires: un pilotage nécessairement relationnel}

Si le pilotage des groupements de similitude reste relativement simple, il en va différemment des groupements complémentaires, où le processus de coopération est beaucoup plus complexe.

\section{Des enjeux multiples nécessitant une véritable coordination cognitive}

Dans le cas des groupements complémentaires, le projet commun consiste à rapprocher les compétences et à créer des synergies entre les partenaires.

Revue internationale P.M.E., vol. 18, $n^{\circ}$ 1, 2005

(C) 2005 - Presses de l'Université du Québec

Édifice Le Delta I, 2875, boul. Laurier, bureau 450, Sainte-Foy, Québec G1V 2M2 - Tél.: (418) 657-4399 - www.puq.ca

Tiré de: Revue internationale P.M.E., vol. 18, $\mathrm{n}^{\circ}$ 1, sous la direction de Louis Raymond • PME1801N

Tous droits de reproduction, de traduction et d'adaptation réservés 


\section{Une coordination technique pluridimensionnelle et complexe}

Lorsqu'elles coopèrent au sein d'un groupement complémentaire, les PME cherchent à toucher de nouveaux marchés par le biais d'une offre globale et/ou d'une innovation commune. Les entreprises tentent d'exploiter conjointement des complémentarités d'actifs tangibles ou intangibles (équipements, connaissances, etc.), en coordonnant leurs activités et réalisent une association pour la réalisation d'un projet spécifique nécessitant une combinaison de ressources et de compétences. Les groupements complémentaires renvoient au développement et à l'utilisation d'une complémentarité mutuelle, en termes de marchés, de produits ou de ressources (incluant les idées). Un groupement complémentaire peut avoir lieu, par exemple, lorsque des firmes spécialisées apportent chacune une composante d'un produit qui est alors vendu au nom du groupement agissant comme une seule entité. Le produit final est donc quelque chose qu' aucune entité n'aurait pu produire seule et qui ne peut être que le résultat d'un processus coopératif ${ }^{19}$.

Les partenaires doivent donc définir les objectifs à atteindre et développer une nouvelle connaissance pour les atteindre. Pour qu' un groupement complémentaire soit un succès, prévenir les risques ne suffit pas; il faut avant tout garantir la réussite du projet commun en facilitant l'apprentissage, l'accès et la création de compétences.

La tâche à réaliser en coopération est nécessairement complexe, car elle implique l'exécution conjointe d'activités de production de ressources par les entreprises partenaires. De fait, le travail collaboratif repose sur la réunion de compétences différentes. Si la réussite du projet passe par la coordination de ces compétences, elle requiert également le développement d'effets d'apprentissage dans le travail commun et la création de compétences collectives. La coordination technique est donc complexe et les mécanismes contractuels ne peuvent y jouer qu'un rôle mineur. En effet, les processus d'apprentissage ne peuvent être décidés ou organisés contractuellement; une incertitude forte subsiste donc quant à leur émergence et à leurs résultats.

19. Une telle coopération offre donc des économies de champ, puisque les différents spécialistes coopérants agissent comme une entité collective et peuvent fournir les clients avec une gamme plus large d'options que celle que pourrait offrir chaque firme individuellement. Mais elle peut également créer des économies d'échelle, car si le volume total de commande augmente, chaque PME peut faire une utilisation plus complète de son équipement.

Revue internationale P.M.E., vol. 18, $\mathrm{n}^{\circ}$ 1, 2005

(C) 2005 - Presses de l'Université du Québec

Édifice Le Delta I, 2875, boul. Laurier, bureau 450, Sainte-Foy, Québec G1V 2M2 - Tél.: (418) 657-4399 - www.puq.ca

Tiré de: Revue internationale P.M.E., vol. 18, $\mathrm{n}^{\circ} 1$, sous la direction de Louis Raymond $\cdot$ PME1801N

Tous droits de reproduction, de traduction et d'adaptation réservés 


\section{L’opportunisme: une menace sérieuse}

La gestion de l'opportunisme semble, elle aussi, plus problématique que dans les groupements de similitude. La coopération est en effet plus risquée, car les entreprises développent généralement des actifs spécifiques qui les placent dans une relation de dépendance bilatérale. Tel est le cas, par exemple, d'une production en complémentarité en vue de fabriquer un produit propre au groupement: des échanges de produits intermédiaires se réalisent entre les différents membres du groupement qui peuvent alors être tentés par des comportements opportunistes, dans la mesure où chacun constitue un maillon essentiel de la chaîne de production du bien et où ses partenaires hésiteront à rompre la relation ${ }^{20}$.

De plus, non seulement des investissements sont spécifiques au groupement, mais on se situe souvent dans le cadre d'une production en équipe. De ce fait, les problèmes de passager clandestin prennent toute leur importance (Alchian et Demsetz, 1972). En effet, le problème de la production en équipe se pose notamment dès qu'interviennent une activité de recherche, une production de connaissances et de compétences communes: s'il est facile de comptabiliser le nombre de pièces produites par telle ou telle firme, ses efforts en matière de recherche, de conception et d'amélioration du produit, de prospection commerciale en vue de chercher de nouveaux clients pour le groupement, etc., sont beaucoup plus difficiles à évaluer. Le succès d'un groupement peut alors reposer sur des chefs d'entreprise plus dynamiques qui tirent l'ensemble vers le haut sans bénéficier pour autant de tous les fruits de leurs efforts. D'autres entreprises en perte de vitesse peuvent voir dans le groupement une opportunité pour accroître leurs connaissances, leur position concurrentielle, sans produire par elles-mêmes tous les efforts nécessaires. Des conflits peuvent en outre survenir lors du partage des bénéfices communs: les contributions individuelles n'étant pas mesurables, des tensions peuvent apparaître lors du partage du surplus, certains pouvant essayer d'en capter une partie au détriment des autres.

L'opportunisme peut en fait se manifester de différentes manières: une entreprise peut ne pas maximiser son effort en fournissant des composants ou un service de qualité inférieure. Elle peut chercher à capter une part plus importante des bénéfices communs en pratiquant des prix de transfert trop élevés. Le risque d'opportunisme est encore plus fort lorsque l'entreprise est située en bout de chaîne, chargée de la commercialisation du produit, en contact avec le client final: dans la mesure où c'est elle qui négocie le prix de vente du bien, elle peut être incitée à

20. Cette dépendance rend le groupement difficilement réversible, car la cessation de la coopération comporte alors un coût extrêmement élevé, voire impossible à assumer pour l'entreprise.

Revue internationale P.M.E., vol. 18, nº 1, 2005

(C) 2005 - Presses de l'Université du Québec

Édifice Le Delta I, 2875, boul. Laurier, bureau 450, Sainte-Foy, Québec G1V 2M2 • Tél.: (418) 657-4399 - www.puq.ca

Tiré de: Revue internationale P.M.E., vol. 18, $\mathrm{n}^{\circ} 1$, sous la direction de Louis Raymond $\cdot$ PME1801N

Tous droits de reproduction, de traduction et d'adaptation réservés 
gonfler ses marges au détriment du groupement. Elle peut chercher à dissimuler des informations qui seraient importantes pour le groupement et à traiter avec certains clients du groupement «en solo».

Mais le risque d'opportunisme est important également à cause du caractère stratégique de la coopération, engendrant un accès aux compétences des partenaires. Ainsi, une firme peut voir dans sa participation au groupement une manière de capter des informations, de profiter d'effets d'apprentissage, de rattraper son retard technologique, de développer une action de veille sur ses concurrents, etc. La firme tire ainsi plus de bénéfices du groupement que ce qu'elle lui apporte. Le risque majeur pour une firme s'engageant dans une telle coopération est alors de se faire déposséder de son savoir-faire par son partenaire, sachant que plus le savoir est codifiable, plus le transfert de compétences est aisé.

Ce risque d'appropriation des compétences d'une firme par un partenaire malhonnête est difficilement maîtrisable par le biais de solutions contractuelles. Si des mécanismes contractuels peuvent être imaginés pour éviter les comportements «classiques» de free riding, d'une part, ces mécanismes sont coûteux et restent faillibles et, d'autre part, ils sont largement inefficaces pour empêcher un transfert non désiré de compétences d'une entreprise vers une autre. Dès lors, dans les groupements complémentaires, la lutte contre l'opportunisme ne peut passer exclusivement par le contrat. De la même manière, si le contrat peut être utile au début de la relation pour assurer une coordination minimale des activités des partenaires, le développement d'effets d'apprentissage suppose la mise en œuvre d'autres mécanismes dans le cours de la relation.

\section{Un pilotage nécessairement relationnel}

Dans les groupements complémentaires, le problème est non seulement de définir ex ante des mécanismes de coordination mais aussi de faciliter l'apprentissage tout au long du processus de coopération. C'est pourquoi un pilotage «relationnel» de ces groupements nous semble plus adapté, fondé non seulement sur le contrat mais surtout sur des mécanismes extra-contractuels développés dans le cours de la relation. Cela ne signifie pas que le contrat de coopération soit inutile; au contraire, quoique incomplet, il est souvent indispensable pour engager la coopération sur des bases solides.

\section{L'engagement: un contrat de coopération incomplet}

À la phase d'engagement, les partenaires vont s'accorder sur un ensemble de mécanismes de base, leur permettant d'entamer leur coopération. Des règles vont spécifier ce que chaque partenaire doit faire dans certaines situations pouvant être facilement prévues ex ante. Par exemple, elles vont déterminer la division du travail

Revue internationale P.M.E., vol. 18, nº 1, 2005

(C) 2005 - Presses de l'Université du Québec

Édifice Le Delta I, 2875, boul. Laurier, bureau 450, Sainte-Foy, Québec G1V 2M2 - Tél.: (418) 657-4399 - www.puq.ca

Tiré de: Revue internationale P.M.E., vol. 18, $\mathrm{n}^{\circ} 1$, sous la direction de Louis Raymond • PME1801N

Tous droits de reproduction, de traduction et d'adaptation réservés 
et l'ordre d'intervention des différentes entreprises dans le processus de production, les modalités de la prospection de nouveaux clients, etc. ${ }^{21}$. Concernant la gestion de l'opportunisme, plusieurs solutions, non mutuellement exclusives, peuvent être envisagées: des mécanismes de surveillance, de rétorsion (échange d'otages par exemple $^{22}$ ) et d'incitation.

La mise en œuvre de la coopération: apprentissage, confiance et pilotage relationnel

Pour que la coopération soit vraiment efficace, les partenaires doivent, dans le cours de leur relation, développer des apprentissages, mais ces apprentissages ne sont pas automatiques. Une coordination informelle est susceptible de les faciliter, en reposant sur une coordination essentiellement hors contrat, construite dans le cours de la relation et fondée notamment sur la confiance et l'ajustement mutuel.

Un groupement complémentaire se justifie par la mise en commun de compétences et ces interrelations sont elles-mêmes créatrices de compétences spécifiques au groupement. La création de compétences n'est pas le seul résultat de la mise en commun de ressources, c'est également le résultat d'un mécanisme particulier de coordination entre des entreprises différentes. Ce processus de coordination nécessite de s'appuyer sur la répétition d'interactions entre les entreprises composant le groupement, c'est-à-dire sur la définition de routines spécifiques au groupement. Nous retrouvons ici l'idée de Nelson et Winter (1982) que l'on peut appliquer à l'analyse des groupements de PME: la coopération donne lieu à des routines de coordination qui fondent la création de compétences spécifiques au groupement, au-delà de la mise en commun des compétences de chaque entreprise. Le «noyau de compétence» d'un groupement représente l'ensemble des apprentissages réalisés dans ce groupement, en particulier des apprentissages relatifs à la coordination des compétences en matière de production. Ces compétences s'améliorent dans l'utilisation, elles peuvent être épargnées, dans une perspective de valorisation des atouts du groupement dans la durée, et surtout, ce sont elles qui permettent au groupement de PME d'accéder à de nouveaux marchés et de se différencier des concurrents.

Or, la confiance mutuelle est souvent considérée dans la littérature comme une condition de l'apprentissage et de la création de compétences organisationnelles au

21. Du fait de la possible apparition d'événements imprévus, le contrat doit également prévoir un mécanisme d'autorité, qui peut prendre la forme d'une assemblée générale, par exemple.

22. Un exemple d'otages est celui des prises de participation croisées au sein des coopérations interentreprises: si l'un des partenaires adopte un comportement opportuniste, les autres peuvent collectivement prendre le contrôle de son entreprise.

Revue internationale P.M.E., vol. 18, nº 1, 2005

(C) 2005 - Presses de l'Université du Québec

Édifice Le Delta I, 2875, boul. Laurier, bureau 450, Sainte-Foy, Québec G1V 2M2 • Tél.: (418) 657-4399 - www.puq.ca

Tiré de: Revue internationale P.M.E., vol. 18, $\mathrm{n}^{\circ} 1$, sous la direction de Louis Raymond $\cdot$ PME1801N

Tous droits de reproduction, de traduction et d'adaptation réservés 
sein des coopérations. Lazaric, par exemple, souligne «l'importance des conditions immatérielles telles que la confiance, la mise en place de connaissances communes ou l'existence de routines, pour l'élaboration d'un apprentissage pendant l'accord» (Lazaric, 1995, p. 134). La confiance apparaît comme un élément renforçant la mise en œuvre de nouvelles pratiques plus performantes. A contrario, l'absence de confiance, sans nécessairement remettre en cause le projet qui rassemble les acteurs, peut limiter les possibilités d'amélioration des performances du groupement. Pour Lundvall (1993), par exemple, l'apprentissage «technique» peut être inhibé lorsque l'apprentissage «social» ou comportemental ne se produit pas.

La confiance dans la capacité de chaque partie à tenir ses engagements peut exister dès l'origine, mais elle demande à être renforcée au cours de l'exécution du contrat (Aliouat, 1996). Plusieurs auteurs proposent ainsi une notion de confiance incrémentale, comme étant construite dans le cours de la relation, lors d'étapes successives ${ }^{23}$. Dans ce cadre, les partenaires ne doivent pas faire un «saut dans l'inconnu», mais procéder par test progressif. Ils doivent d'abord se centrer sur des activités relativement simples, afin que la confiance s'établisse pour des activités plus importantes. La confiance peut ainsi être interprétée comme un cas particulier d'actif spécifique résultant de l'accumulation de comportements passés. Elle est progressivement créée à partir d'une succession d'interactions dans lesquelles la personnalité des participants joue un rôle important. La création de ce «capital relationnel» passe par une forme particulière d'apprentissage interactif, soit l'apprentissage sur le partenaire, dont les bénéfices seraient perdus en cas de cessation de la coopération.

Ainsi, la confiance ne se décrète pas, elle se construit. Plusieurs facteurs peuvent selon nous favoriser cette construction de la confiance au sein des groupements de PME.

Tout d'abord, le contrat de coopération peut être considéré comme un initiateur de la confiance. Cette affirmation peut apparaître a priori comme paradoxale: souvent le contrat est considéré comme la manifestation d'un manque de confiance $^{24}$. Toutefois, plusieurs auteurs notent que le contrat constitue une base pour le développement de la confiance. Pour Brousseau, la passation d'un contrat écrit symbolise un engagement qui témoigne de la volonté de coopérer et permet de créer un climat de confiance, sans lequel la coopération n'est pas possible: «la signature d'un contrat apparaît comme un acte à travers lequel les parties manifestent et matérialisent leur volonté de coopérer» (Brousseau, 1996, p. 46). L'existence d'un contrat de coopération constitue un signe par lequel les parties montrent leur bonne volonté à coopérer et incite chacune d'elles à penser que l'autre va agir dans

23. En particulier Lorenz (1988, 1992), Lazaric et Lorenz (1998).

24. Pour Bradach et Eccles (1991) notamment.

Revue internationale P.M.E., vol. 18, n 1, 2005

(C) 2005 - Presses de l'Université du Québec

Édifice Le Delta I, 2875, boul. Laurier, bureau 450, Sainte-Foy, Québec G1V 2M2 - Tél.: (418) 657-4399 - www.puq.ca

Tiré de: Revue internationale P.M.E., vol. 18, $\mathrm{n}^{\circ} 1$, sous la direction de Louis Raymond P PME1801N Tous droits de reproduction, de traduction et d'adaptation réservés 
le sens de l'intérêt commun, même si des occasions d'opportunisme se présentent. Le contrat apparaît comme initiateur d'une croyance réciproque dans la fiabilité du partenaire. Ainsi, le contrat est indispensable, car il constitue une condition d'émergence de la confiance.

En outre, la construction de la confiance nécessite des interactions entre les partenaires. En effet, dans les travaux sur l'origine de la confiance, l'importance du processus d'interaction entre les parties concernées est très souvent soulignée. Noorderhaven (1996), par exemple, insiste sur le fait que le processus d'interaction influence la confiance des parties à travers l'accumulation d'informations sur la loyauté et la sincérité de l'autre partie. Chaque agent est enrichi par l'information obtenue dans l'ensemble des interactions précédentes et peut modifier son comportement dans le temps. La confiance est donc un processus de connaissance de l'autre et la production de la confiance s'inscrit dans la dynamique des interactions.

Enfin, de manière corollaire, la proximité des partenaires peut favoriser l'émergence de la confiance: la proximité géographique, dans la mesure où elle facilite les interactions, est évidemment importante. Mais l'appartenance des PME à un même territoire est aussi de nature à favoriser l'émergence de la confiance. En particulier, être membre d'une association professionnelle (ou un club d'entrepreneurs, p. ex.) peut fournir, pour des PME, des garanties crédibles contre un comportement opportuniste de ses membres ${ }^{25}$.

Dans un groupement complémentaire, la coopération peut donc être comprise comme un processus incrémental. L'accord débute par la signature d'un contrat qui sera, au fur et à mesure de l'évolution de la coopération, complété par des mécanismes extracontractuels, favorisant et constituant en eux-mêmes des processus d'apprentissage. De nombreux auteurs évoquent la règle du «pas-à-pas», les partenaires devant procéder à des engagements croissants et à une coordination progressivement plus intensive. Pour Lazaric (1999), par exemple, la coopération n'est pas un mécanisme spontané découlant d'une simple volonté des firmes de travailler ensemble: elle résulte d'un long apprentissage collectif où chaque partenaire teste progressivement les compétences et les intentions de l'autre pour juger de sa capacité à respecter un «code de conduite minimal».

\section{Le risque d'évolution des groupements complémentaires vers une forme intégrée}

Si l'apprentissage est nécessaire, il comporte un effet pervers, soit celui d'enfermer progressivement les partenaires à l'intérieur de leur relation. Non seulement le

25. Les institutions peuvent également jouer un rôle important de coordinateur ou de régulateur au sein même des groupements, notamment à l'égard de l'opportunisme.

Revue internationale P.M.E., vol. 18, nº 1, 2005

(C) 2005 - Presses de l'Université du Québec

Édifice Le Delta I, 2875, boul. Laurier, bureau 450, Sainte-Foy, Québec G1V 2M2 • Tél.: (418) 657-4399 - www.puq.ca

Tiré de: Revue internationale P.M.E., vol. 18, $\mathrm{n}^{\circ} 1$, sous la direction de Louis Raymond $\cdot$ PME1801N

Tous droits de reproduction, de traduction et d'adaptation réservés 
groupement devient de plus en plus irréversible, mais il peut également basculer vers une intégration des partenaires. C'est pourquoi la stabilité des groupements complémentaires ne semble généralement pas assurée.

Effectivement, les apprentissages créent une dynamique organisationnelle, à travers laquelle le groupement acquiert des compétences propres. Ces apprentissages, à mesure qu'ils structurent les règles nécessaires à la résolution des conflits et les routines de coordination, conditionnent la trajectoire future du groupement. Au fur et à mesure que progresse la relation, le coût d'une rupture de la coopération devient de plus en plus élevé (du fait des coûts irrécouvrables) et les bénéfices de sa poursuite s'accroissent. Ainsi, les entreprises risquent de devenir de plus en plus dépendantes les unes des autres et du groupement. Le groupement devient alors une forme d'organisation des activités difficilement réversible et peut éventuellement déboucher sur une fusion ${ }^{26}$.

\subsection{Quelques illustrations concrètes}

\section{Tableau 1 \\ Récapitulatif}

\begin{tabular}{lll}
\hline & Groupements de similitude & Groupements complémentaires \\
\hline Engagement & Contrat «complet» & Contrat «incomplet» \\
Réalisation & Règles prescrites & Confiance et apprentissage \\
Cessation & Prévue ex ante & Indéterminée \\
Pilotage & «Contractuel» & «Relationnel» \\
\hline
\end{tabular}

Les deux exemples suivants nous semblent bien illustrer le cadre conceptuel exposé tout au long de cet article ${ }^{27}$.

26. L'évolution vers une forme intégrée représente un risque dans la mesure où les partenaires ne souhaitent pas nécessairement aboutir à cette éventualité. Mais le groupement peut aussi être considéré comme un « mariage à l'essai » (selon la terminologie de Dussauge et Garrette, 1997) avant de resserrer les liens de manière définitive, comme une étape préalable permettant de connaître les potentialités d'un rapprochement futur. Cette question de la fusion nécessiterait sans doute de larges développements, notamment concernant les questions de dépendance et de pouvoir, que nous ne traiterons pas ici.

27. Ces exemples sont issus d'un projet de recherche mené en France dans la région RhôneAlpes entre 1996 et 2003. Pour plus de détails et la présentation d'autres cas, le lecteur pourra se reporter à Peillon (2001).

Revue internationale P.M.E., vol. 18, nº 1, 2005

(C) 2005 - Presses de l'Université du Québec

Édifice Le Delta I, 2875, boul. Laurier, bureau 450, Sainte-Foy, Québec G1V 2M2 • Tél.: (418) 657-4399 - www.puq.ca

Tiré de: Revue internationale P.M.E., vol. 18, $\mathrm{n}^{\circ} 1$, sous la direction de Louis Raymond • PME1801N

Tous droits de reproduction, de traduction et d'adaptation réservés 


\section{Un exemple de groupement de similitude}

Le premier exemple concerne un groupement créé en 1997 par cinq PME travaillant dans des secteurs différents, qui ont décidé de se regrouper, car elles désiraient toutes obtenir la certification qualité ISO 9000, alors qu' aucune n' avait, individuellement, les moyens de le faire. Le groupement a donc été créé, sous la forme d'un groupement d'employeurs, afin d'embaucher un qualiticien en temps partagé et d'obtenir la certification pour l'ensemble des membres, en en minimisant le coût.

Ce groupement peut être qualifié de groupement de similitude intersectoriel et son fonctionnement nous semble révélateur d'un pilotage contractuel.

L'objectif (la certification 1SO 9000) et les moyens mis en œuvre pour l'atteindre (l'embauche du qualiticien) sont définis dès le début de la relation. Pour réaliser leur objectif, les partenaires ont créé une association dont les statuts et le règlement intérieur peuvent être considérés comme représentant le contrat de coopération. Ce contrat peut être considéré comme «complet»: les statuts et le règlement intérieur prévoient quasiment tous les aspects du fonctionnement du groupement. Un mécanisme d'autorité a été défini, sous la forme d'un conseil d'administration, dont la composition et le rôle sont spécifiés dans les statuts de l'association. Des routines contractuelles ont été créées: le règlement intérieur précise la rémunération, les objectifs de la mission et l'emploi du temps du qualiticien, qui répartit ses interventions dans chacune des entreprises de manière égale.

Le risque d'opportunisme réside dans une possible «surexploitation» du salarié commun. Mais, son emploi du temps étant défini par une règle contractuelle et aucun membre ne pouvant déroger à cette règle sans que les autres en soient très rapidement informés, ce risque est en réalité limité. Une autre forme de comportement opportuniste consisterait, pour certains membres, à profiter du regroupement sans en supporter les coûts. Cette possibilité a néanmoins été anticipée, puisque la radiation de l'association est prévue par les statuts en cas de non-paiement de la cotisation annuelle par l'un des membres.

En 2000, les entreprises ont toutes obtenu la certification ISO 9000. Le qualiticien a rempli son mandat dans une fourchette de coût acceptable pour les entreprises et la démarche a été moins coûteuse que si elle avait été assumée par un consultant. Les bénéfices du regroupement ne sont donc pas négligeables pour ces entreprises. On peut également considérer que la cessation de la coopération a été envisagée dès le départ, puisque le statut juridique a été choisi du fait des facilités de dissolution et d'entrée et sortie des membres.

Au total, le pilotage de la relation peut être considéré comme complètement défini ex ante.

Revue internationale P.M.E., vol. 18, $n^{\circ}$ 1, 2005

(C) 2005 - Presses de l'Université du Québec

Édifice Le Delta I, 2875, boul. Laurier, bureau 450, Sainte-Foy, Québec G1V 2M2 • Tél.: (418) 657-4399 - www.puq.ca

Tiré de: Revue internationale P.M.E., vol. 18, $\mathrm{n}^{\circ} 1$, sous la direction de Louis Raymond $\cdot$ PME1801N

Tous droits de reproduction, de traduction et d'adaptation réservés 


\section{Un exemple de groupement complémentaire}

Le second exemple concerne un groupement créé en 1999 par trois PME complémentaires du secteur électronique. L'objectif est de développer une activité propre en proposant une offre complète de savoir-faire et de services, allant de la conception à la fabrication en série de matériels électroniques, sur les marchés des télécommunications et du ferroviaire. Ainsi, il s'agit typiquement d'un groupement complémentaire vertical, dans lequel des PME, habituellement dans des relations de clients/fournisseurs coopèrent autour d'activités étroitement complémentaires. Un technico-commercial a en outre été recruté pour démarcher les clients dans des régions ciblées.

Dans le cas de ce groupement, peu de mécanismes contractuels semblent avoir été définis ex ante, que ce soit au plan de la coordination technique ou de la prévention de l'opportunisme.

Au regard de la coordination technique, seule une règle prévoyant que les démarches du commercial soient, au début, réalisées avec au moins un dirigeant des trois sociétés et un programme de participation à des salons et à des conventions d'affaires ont été décidés. Ce faible recours à des mécanismes contractuels peut s'expliquer par le fait que les trois entreprises travaillaient régulièrement ensemble depuis plusieurs années et que des mécanismes de coordination existaient déjà, avant même la création du groupement.

En ce qui a trait à la lutte contre l'opportunisme, rien ne semble avoir été prévu. Les entreprises sont liées financièrement, mais seulement pour une petite part de capital et seule une structure juridique prenant la forme d'une association a été créée. L'absence de mécanismes de lutte contre l'opportunisme peut s'expliquer par une connaissance antérieure de longue date des dirigeants des entreprises; le temps et les expériences professionnelles communes leur ont permis de se connaître, de se faire confiance, de s'apprécier techniquement et humainement. La logique de filière métier a également pu contribuer au rapprochement «naturel» des entreprises, qui bénéficient d'une solide complémentarité technique. De plus, l'importance de l'enjeu de cette coopération, les dirigeants étant convaincus que le développement de leur entreprise passe par une action concertée, rend les comportements opportunistes moins attrayants. Néanmoins, les membres du groupement envisagent de choisir une autre structure juridique afin de «responsabiliser les partenaires dans les projets communs» et de permettre «la gestion et la définition des responsabilités dans les actions communes». Ainsi, une formalisation de la coopération, avec des garanties contractuelles, semble nécessaire, malgré la connaissance et la confiance mutuelle qui existent entre les partenaires.

Concernant la mise en œuvre de la coopération, un apprentissage organisationnel est largement perceptible. En effet, les premières affaires réalisées en

Revue internationale P.M.E., vol. 18, nº 1, 2005

(C) 2005 - Presses de l'Université du Québec

Édifice Le Delta I, 2875, boul. Laurier, bureau 450, Sainte-Foy, Québec G1V 2M2 • Tél.: (418) 657-4399 - www.puq.ca

Tiré de: Revue internationale P.M.E., vol. 18, $\mathrm{n}^{\circ} 1$, sous la direction de Louis Raymond • PME1801N

Tous droits de reproduction, de traduction et d'adaptation réservés 
commun ont permis d'améliorer l'organisation de la coopération, impliquant à la fois les services commerciaux et les services techniques des différentes entreprises. Et même si peu de mécanismes contractuels ont été définis ex ante, après les premières affaires traitées conjointement, des règles établissant les responsabilités et les engagements de chacun ont été formulées. Ainsi, une période «transitoire» a permis de tester et d'améliorer l'organisation des relations.

Enfin, la cessation de la coopération n'est pas envisagée. Néanmoins, aucune compétence technique propre au groupement n'ayant encore été créée, comme par exemple une innovation produit, la coopération ne semble pas encore irréversible.

\section{Conclusion}

Au total, si les groupements complémentaires nous paraissent présenter davantage d'opportunités pour les PME que les groupements de similitude, ils engendrent cependant des risques plus élevés, plus difficilement prévisibles et donc plus difficiles à contourner par des contrats adéquats. Dans les groupements complémentaires, d'une part, la tâche devant être réalisée en coopération est plus complexe que dans les groupements de similitude et nécessite une forte coordination et, d'autre part, le risque d'opportunisme y est aussi plus saillant. C'est pourquoi si l'efficacité des groupements de similitude peut être assurée grâce à des règles contractuelles, celle des groupements complémentaires, en revanche, nécessite la mise en œuvre de processus d'apprentissage dans le cours de la relation. Un pilotage relationnel, basé sur une confiance solide, de nombreuses interactions et un ajustement mutuel entre les partenaires est dès lors le mieux adapté pour parvenir aux résultats escomptés.

Pour reprendre les propos de Favereau (1989), dans le cas des groupements complémentaires, «l'incomplétude n'est pas le problème, c'est la solution». En effet, ce qui pouvait paraître une contrainte constitue, en fait, une opportunité pour les entreprises partenaires: l'incomplétude va faciliter l'apprentissage au sein de la coopération. Foss (1996) développe toute une argumentation en ce sens-là qui concerne la firme, mais que l'on peut étendre à la coopération interfirmes: si l'objectif de la coopération est la création de connaissances, de «nouvelles options», cela requiert des processus d'apprentissage organisationnel qui vont, en eux-mêmes, créer des événements imprévus. En conséquence, pour le processus de création de connaissances, l'incomplétude contractuelle semble nécessaire. Du fait de l'incertitude, les contrats sont forcément incomplets, les parties étant incapables de fixer dès le départ l'ensemble des états de la nature et les solutions appropriées. C'est donc l'incomplétude des contrats qui va susciter un besoin de coordination relationnelle entre les partenaires pour pallier l'insuffisance contractuelle et réaliser 1 'apprentissage.

Revue internationale P.M.E., vol. $18, \mathrm{n}^{\circ} 1,2005$

(C) 2005 - Presses de l'Université du Québec

Édifice Le Delta I, 2875, boul. Laurier, bureau 450, Sainte-Foy, Québec G1V 2M2 - Tél.: (418) 657-4399 - www.puq.ca

Tiré de: Revue internationale P.M.E., vol. 18, $\mathrm{n}^{\circ} 1$, sous la direction de Louis Raymond $\cdot$ PME1801N

Tous droits de reproduction, de traduction et d'adaptation réservés 
Cet article propose un cadre conceptuel général d'analyse de la coopération entre PMI. Cette contribution nous semble importante dans la mesure où la coopération entre PME avait jusqu'à présent été davantage analysée à travers les concepts de district industriel ou de système productif local. Cependant, même si deux exemples concrets ont été présentés, qui semblent aller dans le sens de l'argumentation développée, ce cadre théorique reste encore à valider, notamment par d'autres études de cas.

\section{Bibliographie}

Alchian, A.A. et H. Demsetz (1972), «Production, information costs and economic organization», American Economic Review, vol. 62, n 5, p. 777-795.

Aliouat, B. (1996), Les stratégies de coopération industrielle, Paris, Economica.

BRADACH, J.L. et R.G. ECCLES (1991), «Price, authority and trust: from ideal types to plural forms», dans G. Thompson et al. (dir.), Markets, Hierarchies and Networks. The Coordination of Social Life, Londres, Sage Publications, p. 277-292.

Brousseau, E. (1993), L'économie des contrats: technologies de l'information et coordination interentreprises, Paris, Presses universitaires de France.

Brousseau, E. (1996), «Les contrats dans la coordination interentreprises: les enseignements de quelques travaux récents d'économie appliquée», dans T. Andreani (dir.), L'entreprise, lieu de nouveaux contrats, Paris, L'Harmattan.

Doz, Y.L. (1996), «The evolution of cooperation in strategic alliances: initial conditions or learning processes?», Strategic Management Journal, $\mathrm{n}^{\circ}$ 17, p. 55-83.

Dussauge, P. et B. Garrette (1997), «Anticiper les conséquences des alliances stratégiques», Revue française de gestion, $\mathrm{n}^{\circ} 114$, p. 106-117.

Duysters, G.M., A.P. DE MAn et L. Wildeman (1999), «A network approach to alliance management», European Management Journal, vol. 17, nº 2, p. 182-187.

Duysters, G.M. et K.H. HeIMERIKs (2002), «Alliance capabilities - how can firms improve their alliance performance?», 6th International Conference on Competence-based Management, IMD Lausanne, 26-28 octobre.

FAVEREAU, O. (1989), «Marchés internes, marchés externes», Revue économique, vol. 40, $\mathrm{n}^{\circ} 2$, p. 273-328.

Foss, N.J. (1996), «Firms, incomplete contracts and organizational learning», Human System Management, $\mathrm{n}^{\circ} 15$, p. 17-22.

Joffre, P. et G. KoENIG (1984), «Stratégies de coopération et d'alliances inter-entreprises», Enseignement et Gestion, $\mathrm{n}^{\circ}$ 31, p. 67-73.

LAZARIC, N. (1995), «Apprentissage organisationnel et accords inter-firmes: le cas de la relation utilisateur/producteur», dans N. Lazaric et J.M. Monnier (dir.), Coordination économique et apprentissage des firmes, Paris, Economica, p. 133-154.

LAZARIC, N. et E. LoREnZ (dir.) (1998), Trust and Economic Learning, Londres, Edward Elgar.

Revue internationale P.M.E., vol. 18, nº 1, 2005

(C) 2005 - Presses de l'Université du Québec

Édifice Le Delta I, 2875, boul. Laurier, bureau 450, Sainte-Foy, Québec G1V 2M2 - Tél.: (418) 657-4399 - www.puq.ca

Tiré de: Revue internationale P.M.E., vol. 18, no 1, sous la direction de Louis Raymond PME1801N

Tous droits de reproduction, de traduction et d'adaptation réservés 
LAZARIC, N. (1999), «Apprentissage collectif, compétences et nouvelle division du travail quels enjeux et quels débats pour la coopération», Colloque «Coopération industrielle diversité et synthèse», ADIS, Faculté Jean-Monnet, Université Paris Sud, 3-4 mai.

Llerena, P., M. Matt et S. WolfF (1999), «Incentives and knowledge creation in cooperative inter-organization agreements», Colloque «Coopération industrielle: diversité et synthèse», ADIS, Faculté Jean-Monnet, Université Paris Sud, 3-4 mai.

LORENZ, E.H. (1988), «Neither friends nor strangers: informal networks of subcontracting in French industry», dans D. Gambetta (dir.), Trust, Making and Breaking Cooperative Relations, Oxford, Basil Blackwell, p. 195-210.

LORENZ, E.H. (1992), «Trust, community and cooperation: toward a theory of industrial districts», dans M. Storper et A. Scott (dir.), Pathways to Industrialization and Regional Development, Londres, Routledge, p. 195-204.

LuNDVALL, B.A. (1993), «Explaining interfirm cooperation and innovation. Limits of the transaction-cost approach», dans G. Grabher (dir.), The Embedded Firm: On the Socio-Economics of Industrial Networks, Londres, Routledge, p. 52-64.

Nelson, R.R. et S.G. Winter (1982), An Evolutionary Theory of Economic Change, Cambridge, Mass., Harvard University Press.

NoORderhaVEN, N.G. (1996), «Opportunism and trust in transaction cost economics», dans J. Groenewegen (dir.), Transaction Cost Economics and Beyond, Londres, Kluwer Academic Publishers, p. 105-128.

ORGANISATION DE COOPÉRATION ET DE DÉVELOPPEMENT ÉCONOMIQUES - OCDE (1996), Réseaux d'entreprises et développement local. Compétition et coopération dans les systèmes productifs locaux, Paris, Éditions de l'OCDE.

PeILlon, S. (2001), Le pilotage des coopérations interentreprises: le cas des groupements de $P M E$, Thèse pour le doctorat de sciences économiques, École nationale supérieure des mines de Saint-Étienne, Université Jean-Monnet, 3 octobre.

PopPo, L. et T. ZENGER (2002), «Do formal contracts and relational governance function are substitutes or complements?», Strategic Management Journal, vol. 23, p. 707-725.

RICHARDSON, G.B. (1972), «The organization of industry», The Economic Journal, $\mathrm{n}^{\circ}$ 82, septembre, p. 883-896.

RING, P.S. et A.H. VAN DE VEN (1994). «Developmental processes of cooperative interorganizational relationships», Academy of Management Review, vol. 19, n 1, p. 90-118.

WeInstein, O. (1997), «Modes de coordination interfirmes et modèles d'innovation», dans C. Palloix et Y. Rizopoulos (dir.), Firmes et économie industrielle, Paris, L'Harmattan, p. 389-413.

Revue internationale P.M.E., vol. $18, \mathrm{n}^{\circ} 1,2005$

(C) 2005 - Presses de l'Université du Québec

Édifice Le Delta I, 2875, boul. Laurier, bureau 450, Sainte-Foy, Québec G1V 2M2 - Tél.: (418) 657-4399 - www.puq.ca

Tiré de: Revue internationale P.M.E., vol. 18, $\mathrm{n}^{\circ}$ 1, sous la direction de Louis Raymond • PME1801N

Tous droits de reproduction, de traduction et d'adaptation réservés 